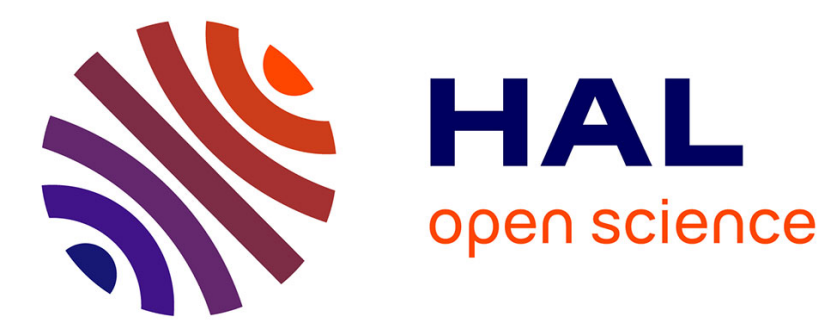

\title{
Crystal plasticity parameter identification with 3D measurements and Integrated Digital Image Correlation
}

Morgan Bertin, Chaowei Du, Johan P M Hoefnagels, François Hild

\section{To cite this version:}

Morgan Bertin, Chaowei Du, Johan P M Hoefnagels, François Hild. Crystal plasticity parameter identification with 3D measurements and Integrated Digital Image Correlation. Acta Materialia, 2016, 116, pp.321 - 331. 10.1016/j.actamat.2016.06.039 . hal-01383887

\section{HAL Id: hal-01383887 \\ https://hal.science/hal-01383887}

Submitted on 19 Oct 2016

HAL is a multi-disciplinary open access archive for the deposit and dissemination of scientific research documents, whether they are published or not. The documents may come from teaching and research institutions in France or abroad, or from public or private research centers.
L'archive ouverte pluridisciplinaire HAL, est destinée au dépôt et à la diffusion de documents scientifiques de niveau recherche, publiés ou non, émanant des établissements d'enseignement et de recherche français ou étrangers, des laboratoires publics ou privés. 


\title{
Crystal plasticity parameter identification with 3D measurements and Integrated Digital Image Correlation
}

\author{
Morgan Bertin ${ }^{1}$, Chaowei Du ${ }^{2}$, Johan P.M. Hoefnagels ${ }^{2}$, and François Hild ${ }^{1}$ \\ ${ }^{1}$ LMT (ENS Cachan, CNRS, Université Paris-Saclay) \\ 61 avenue du Président Wilson, 94235 Cachan, France \\ Email: \{bertin,hild\}@lmt.ens-cachan.fr \\ ${ }^{2}$ TUE (Technische Universiteit Eindhoven) \\ 5612 AZ Eindhoven, Netherlands \\ Email: $\{$ C.Du,J.P.M.hoefnagels\}@tue.nl
}

\begin{abstract}
The present study unravels details of the micromechanical behavior of a microspecimen made of IF-steel. A triangular prism is machined via focused ion beam (FIB) and contains two ferritic grains. Four experimental tools are integrated to identify the material's crystal parameters: $i$ ) an optical confocal microscope captures height profile images, $i i$ ) an in-situ tensile stage prescribes the loading history to the macro specimen, $i i i)$ a global Digital Image Correlation (DIC) algorithm measures the 3D surface displacement fields, and $i v$ ) an extension of Integrated-DIC for 3D displacement fields is implemented to assess the micromechanical behavior. It is demonstrated that with this methodology the identification of the boundary conditions and crystal plasticity parameters is successfully achieved.
\end{abstract}

Keywords: Crystal plasticity; Digital image correlation; Finite-element

*Corresponding author. François Hild, Email: hild@lmt.ens-cachan.fr, Fax: +33 14740 2240

Preprint submitted to Acta Materialia

March 8, 2016 
analysis; In-stu test; Optical microscopy

\section{Introduction}

The mechanical properties of materials often originate from physical and multi-scale phenomena that are due to complex and heterogenous microstructures. One of the first method to consider heterogeneous media for computing macromechanical responses is based on homogenization [1. In order to solve this problem micromechanical frameworks have been developed for elastic materials [2] and in elastoplasticity [3]. Due to complex behaviors and the difficulty to observe and measure at the microscale the main difficulty remains the identification of the constitutive parameters.

The lattice structure (e.g., FCC, BCC) is considered to derive the constitutive equations for single crystals [4, 5, 6]. The present study aims at coupling experimental and numerical results and provides a compromise between a reasonable computation time and a sufficiently small scale to achieve the observation of the micromechanical behavior in a material with real engineering applications. Under the same assumptions, Raabe et al. 7] characterized the micromechanical behavior in polycrystalline aluminum with the measurement of the displacement on the observe surface. The authors demonstrated by 2-dimensional Finite Element analyses that the micromechanical response obtained from the crystal plasticity law was closer to the experimental observation and was able to predict the stress and strain heterogeneities. However, the underlying microstructure is a key feature and 3D orientation mapping has 
been performed by Musienko et al. [8] to compare experimental and numerical strains also on polycrystalline aluminum. Using a 3D analysis, Martins et al. 9] focused on the residual strain measured in a weld zone of 316L stainless steel.

Digital Image Correlation enables full displacement and strain fields to be measured and provides insight into the micromechanics of solids. Imaging systems capable of higher magnifications allow the heterogeneity of the micromechanical response to be evaluated. A first approach [10] using full field measurements obtained from SEM acquisitions has led to the identification of a single crystal plasticity law. Dmitrieva et al. [11] studied shear bands in a single copper crystal and Tasan et al. [12] characterized micromechanical banding effects. Other techniques may be used, e.g., Raabe et al. [13] acquired pictures with a confocal microscope.

The combination of both experimental and numerical aspects has been investigated by Héripré et al. 14] while applying the Finite Element Method Updating technique (FEMU [15]). Tasan et al. [16] proposed an integrated experimental-numerical approach to investigate stress-strain partitioning in multiphase alloys, although, the mechanical properties of the material have been obtained with a separated technique, i.e., nano indentation tests. Guery et al. [17] have identified constitutive parameters of the law proposed by Méric and Cailletaud [18] using full field measurement, SEM acquisitions and FEMU-UF. The results provide an internal check of the constitutive model with respect to the experiment with the identified parameters. However, several difficulties remain (e.g., unknown underlying microstructure) and the specimen design is still one 
of the biggest challenges because it is hard to build and to test mechanically.

The objective of this study is to perform an investigation at the microscale and to identify the material parameters of a crystal plasticity model with Integrated Digital Image Correlation (IDIC [19, 20, 21, 22]). Figure 1 shows the designed identification procedure. Initially, the crystallographic features, i.e., phase, grain (size, number and orientation) and the geometry of the studied volume are assessed and lead to the design of a representative 3D numerical model. Secondly, the out-of-plane displacement holds essential information regarding the active slip systems. Therefore an experimental method is introduced that utilizes confocal microscopy combined with a 3D surface global DIC formulation to quantify full three-dimensional displacement field of the specimen surface and use these 3D data to extract boundary conditions and identify crystal plasticity parameters. Because of the relatively height noise level computations are run with stabilized boundary conditions. Finally, the sought parameters are identified with integrated-DIC that combines all experimental data, i.e., the surface height profiles, constitutive model, and crystallographic features.

The paper is organized as follows. Section 2 introduces the experimental and numerical tools utilized herein. Section 3 shows the experimental results prior to the identification discussed in Section 4.

\section{Coupled experimental/numerical procedure}

The objectives are $i$ ) to observe a crystal-specific micromechanical behavior under confocal microscopy, ii) to quantitatively measure the 3D surface dis- 
placement field at the grain scale with DIC, iii) to simulate the mechanical response with a representative model of the microstructure and the chosen crystal plasticity law, and $i v$ ) to couple experimental and numerical results in a single numerical framework to identify (as much as possible) the material and kinematic unknowns. The experimental methodology relies on the measurement of surface height profiles of a micro-specimen ( $\mu$-specimen) within a macroscopic sample that is mechanically loaded in tension.

\subsection{Specimen}

To demonstrate the potential of the proposed technique a relevant engineering material is chosen for this study, namely, 1-mm thick as-received IF-steel sheet. The latter possesses a very low carbon content and Table 1 gathers its chemical composition. IF steel is widely used, e.g., in the automotive industry, for its deep drawability properties [23].

The desired mechanical properties of IF-steels are, $a$ ) low yield stress, $b$ ) high hardening modulus resulting in stress levels after the material has been plastically deformed, $c$ ) high Lankford coefficients to improve deep-drawability capabilities and $d$ ) high fracture strain. The ferritic microstructure is body center cubic (BCC). The average grain size is of the order of $10 \mu \mathrm{m}$.

The preparation of the specimen requires four steps:

$i$. From the $1-\mathrm{mm}$ thick sheet, a large specimen is cut $(50 \mathrm{~mm} \times 10 \mathrm{~mm})$ and ground down to $0.3 \mathrm{~mm}$ thickness and polished on both sides, to yield a very flat specimen with the two surfaces parallel to each other. 
ii. A large surface area of the large specimen is investigated using Orientation Imaging Microscopy (OIM, see Figure 2(a,b)). The location of the $\mu$ specimen is chosen to contain two grains that are larger than $15 \mu \mathrm{m}$ having a clean grain boundary and a misorientation angle less than $1^{\circ}$ within the grain so that both crystal and structure are relatively clean (Figure 2(c)).

iii. The $\mu$-specimen is milled using Focused Ion Beam technique (Figure2(d)). The obtained $\mu$-specimen is $12 \mu \mathrm{m}$ long and $4 \mu \mathrm{m}$ wide with an equilateral triangular basis. Its length and depth are a compromise between a size consistent with the resolution of confocal microscopy and the milling speed of the FIB process.

$i v$. The last step is platinum deposition to create a random pattern for DIC purposes. The latter is obtained by depositing $20 \mathrm{~nm}$ high and $0.8 \mu \mathrm{m}$ in diameter semi-spherical dots at specific locations. Their size has been adapted with respect to the confocal microscope features.

Figure 2 shows secondary electron images related to steps $(i i i)$ and $(i v)$. Figure 2(d) shows the underlying microstructure and the grain boundary by observation from the side. Furthermore, it validates the assumption that the apex line of the $\mu$-specimen is entirely free. Figure 2 (e) shows the pattern obtained after platinum deposition. The latter has also been deposited on the border of the $\mu$-specimen to perform DIC on a larger area.

Table 2 gathers the lattice orientations of the two grains identified from the OIM analysis. 


\subsection{Experimental protocol}

Figure 3 shows the experimental apparatus within the confocal microscope, i.e., the large specimen clamped in the tensile stage. Before starting the experiment, the $\mu$-specimen is successively, $i$ ) positioned and aligned inside the tensile stage, $i i$ ) aligned with the translation and rotation stage, and iii) positioned in the center of the field of view of the sensor. During the experiment the large specimen is subjected to a velocity equal to $0.5 \mu \mathrm{m} . \mathrm{s}^{-1}$ and at approximatively every $5 \mu \mathrm{m}$ stopped while the image acquisition procedure is performed. Because the early stage of plastic strain and its corresponding kinematic hardening behavior are being sought, three cycles corresponding to $1 \%, 2 \%$ and $3 \%$ global strain are applied without compression.

Since the $\mu$-specimen can never be perfectly centered on the microscale it moves in the field of view when strained. Therefore, an in-house routine was written to maintain the $\mu$-sample inside the field of view by correcting the rigid body motions using DIC and controlling the motorized $(x, y)$-sample accordingly.

The first image of the experiment corresponds to the reference, then for each load step, a first image is captured to evaluate the rigid body motions. The latter ones are applied as a correction by moving the motorized $(x, y)$-stage and finally a second image is captured. In the $z$-direction, the contraction of the large specimen will move the $\mu$-sample out of the depth of field. An auto-frontal procedure is used to address this issue.

Height profile images are captured in confocal profilometry mode by a Sen- 
sofar $\mathrm{Pl} \mu 2300$ Optical Confocal Microscope using a CCD camera (definition: $557 \times 557$ pixels) with a Nikon EPI $150 \times$ objective lens, resulting in a square field of view of $61 \times 61 \mu \mathrm{m}^{2}$. This technique allows for a very high resolution in height (estimated to be $9 \mathrm{~nm}$ for this particular specimen configuration) whereas the in-plane resolution is limited by the diffraction limit of the blue light wavelength. However, a careful inspection of the height profiles revealed some artifacts caused by, $i$ ) the lens defects and dust particles, and $i i$ ) the CCD pixel positions. Both errors were identified to be systematic because they remain at the same location on the images as long as the imaging parameters are fixed and the same lens is used.

Figure 4(a) shows an image with the systematic errors previously listed. The correction method consists of an acquisition under the identical parameters of 400 images at 400 random locations on a flat reference surface. All images are averaged, i.e., added and normalized by the total number of images, and a plane is fitted and subtracted to this average profile to obtain a mean height level of zero mean with the corresponding root mean square error in the height level as small as possible. This process allows only the systematic error to be kept (Figure 4(b)) and by subtraction from all the experimental images the biases are almost completely removed without corrupting the experimental data. This procedure helps the DIC computation that needs reliable height levels over time. The loads are also measured and the standard resolution is evaluated as $\gamma_{F}=1 \mathrm{~N}$ 


\subsection{D Digital Image Correlation}

Global DIC 24] relies on the registration of an image $f$ in the reference configuration and a series of pictures $g$ in the deformed configurations. The specific approach followed herein adds to the conventional 2D approach the out-of-plane displacement as an offset to the height conservation [25, 26, 27. The registration problem consists of minimizing the sum of squared differences between the deformed image corrected by the measured 3D displacements $\boldsymbol{u}(\boldsymbol{x}, t)=u(\boldsymbol{x}, t) \boldsymbol{e}_{x}+v(\boldsymbol{x}, t) \boldsymbol{e}_{y}+w(\boldsymbol{x}, t) \boldsymbol{e}_{z}$ and the reference image (written for each time $t$ independently) over the Region of Interest

$$
\left.\chi_{f}^{2}(t)=\frac{1}{2 \gamma_{f}^{2} N_{\Omega}} \sum_{\Omega} g\left(\boldsymbol{x}+u(\boldsymbol{x}, t) \boldsymbol{e}_{x}+v(\boldsymbol{x}, t) \boldsymbol{e}_{y}, t\right)-f(\boldsymbol{x}, t)-w(\boldsymbol{x}, t) \boldsymbol{e}_{z}\right)^{2}
$$

with respect to the parameterization of the sought displacement fields $\boldsymbol{u}(\boldsymbol{x}, t)$, where $\boldsymbol{x}=x \boldsymbol{e}_{x}+y \boldsymbol{e}_{y}$ is any considered pixel, $\Omega$ the Region of Interest (ROI), $N_{\Omega}$ its area in terms of number of pixels. The term $\gamma_{f}$ represents the standard deviation (expressed in height level) of noise (assumed additive white Gaussian) that affects each image independently (including the reference one, which is responsible for the factor of 2). Ten images have been acquired initially and the standard deviation is evaluated to be $\gamma_{f}=9 \mathrm{~nm}$. The minimization of $\chi_{f}^{2}$ is achieved by successive linearizations and corrections, using a modified Newton Raphson scheme

$$
[\boldsymbol{M}]\{\delta \boldsymbol{u}\}=\left\{\boldsymbol{b}^{(i)}\right\}
$$

where $[\boldsymbol{M}]$ is the DIC matrix, $\{\delta \boldsymbol{u}\}$ the vector gathering all increments of measured displacement amplitudes, and $\left\{\boldsymbol{b}^{(i)}\right\}$ the residual column vector at itera- 
tion $i$. The DIC matrix reads

$$
M_{i j}=\sum_{\Omega}\left(\nabla f(\boldsymbol{x}) \cdot \boldsymbol{\psi}_{i}(\boldsymbol{x})\right)\left(\boldsymbol{\nabla} f(\boldsymbol{x}) \cdot \boldsymbol{\psi}_{j}(\boldsymbol{x})\right)
$$

and the right hand side term

$$
b_{i}=\sum_{\Omega}\left(\boldsymbol{\nabla} f(\boldsymbol{x}) \cdot \boldsymbol{\psi}_{i}(\boldsymbol{x})\right)(f(\boldsymbol{x})-g(\boldsymbol{x}+\tilde{\boldsymbol{u}}(\boldsymbol{x})))
$$

where $\tilde{\boldsymbol{u}}(\boldsymbol{x})$ is the current estimate of the displacement field and the image gradient is enriched with the variations of height levels, namely, $\boldsymbol{\nabla} f=\frac{\partial f}{\partial x} \boldsymbol{e}_{x}+$ $\frac{\partial f}{\partial y} \boldsymbol{e}_{y}-\boldsymbol{e}_{z}$. A consequence of the addition of the out-of-plane displacement in the functional is that the problem has more degrees of freedom that might affect the minimization.

The integration of mechanical and kinematic constraints into the 3D-DIC problem, namely Integrated-DIC (IDIC), is performed by choosing as unknowns the sought parameters associated with a constitutive law or boundary conditions. The sensitivity fields $\boldsymbol{S}_{U}(\boldsymbol{x})[28$ are chosen as a basis of functions for the displacement field

$$
\left[\boldsymbol{S}_{\boldsymbol{U}}\right]=\frac{\partial\{\boldsymbol{u}\}}{\partial\{\boldsymbol{p}\}}
$$

where $\{\boldsymbol{u}\}$ is the computed nodal displacement field, $\{\boldsymbol{p}\}$ is the vector gathering the unknown parameters, i.e., sought material parameters or boundary conditions. Furthermore, specific features of the loading history may or may not be sensitive. Treating an image series is equivalent to summing over the corresponding contributions at each time step: $\bar{\chi}_{f}^{2}=1 / N_{t} \sum_{t=1}^{N_{t}} \chi_{f}^{2}(t)$ to probe the sensitivities over the whole time domain. The solution at convergence gives an evaluation of the model error compared to the noise level. This indicator is 
useful because it allows the user to validate or not the investigated constitutive $\operatorname{law}(\mathrm{s})[21$.

Last, if the same mesh is used, and the DIC matrix has been computed IDIC simply consists of projecting the nodal displacement field onto the sensitivity fields [29]. Thus, the sought parameters are identified by iteratively solving the linear system until convergence

$$
\{\delta \boldsymbol{p}\}=\frac{1}{2 \gamma_{f}^{2}}[\boldsymbol{M}]_{I D I C}^{-1}\left[\boldsymbol{S}_{\boldsymbol{U}}\right]^{t}\{\boldsymbol{b}\}
$$

where $[\boldsymbol{M}]_{I D I C}=1 /\left(2 \gamma_{f}^{2}\right)\left[\boldsymbol{S}_{\boldsymbol{U}}\right]^{t}[\boldsymbol{M}]\left[\boldsymbol{S}_{\boldsymbol{U}}\right]$ is the kinematic Hessian. The covariance matrix of the identified parameters is written as [30, 21]

$$
\left[\boldsymbol{C}_{p}^{I}\right]=\langle\{\delta \boldsymbol{p}\} \otimes\{\delta \boldsymbol{p}\}\rangle=[\boldsymbol{M}]_{I D I C}^{-1}
$$

where $\langle\bullet\rangle$ is the mean value of $\bullet$. Another useful indicator is the correlation matrix (no index summation used), where $i$ refers to the row number and $j$ the column number of the sought parameter

$$
\left(C o r_{p}\right)_{i j}=\frac{\left(C_{p}\right)_{i j}}{\sqrt{\left(C_{p}\right)_{i i}\left(C_{p}\right)_{j j}}}
$$

\subsubsection{Crystal plasticity model}

To demonstrate the potential of the proposed technique to identify parameters of a crystal plasticity model, the Méric and Cailletaud [18] model is chosen. It is based on the decomposition of the total strain into an elastic and plastic strain. The elastic behavior assumes cubic symmetry in agreement with the BCC lattice. Using the Kelvin-Voigt notations, Hooke's fourth-order tensor [C] 
depends on three elastic constants of the material, namely $C_{11}, C_{12}$ and $C_{44}$. The plastic deformation initiates from crystallographic slip in the crystal lattice and satisfies incompressibility. A slip system $(\theta)$ is defined by a slip plane $\boldsymbol{n}_{0}^{\theta}$ and a slip direction on the plane $s_{0}^{\theta}$ in the initial configuration of the lattice $\left(\bullet_{0}\right)$. In the case of a BCC crystallographic lattice, 24 slip systems are considered, namely 12 principal slip systems from $\langle 110\rangle$ and 12 secondary systems from $\langle 112\rangle$. The gradient tensor $\boldsymbol{F}^{\theta}=\nabla_{0}^{t} \otimes \boldsymbol{x}$, where $\otimes$ is the dyadic product, corresponds to the lattice strain

$$
\boldsymbol{F}^{\theta}=\boldsymbol{I}+\gamma^{\theta} \boldsymbol{s}_{0}^{\theta} \otimes \boldsymbol{n}_{0}^{\theta}
$$

where $\gamma^{\theta}$ is the applied shear on the $\theta^{\text {th }}$ slip system, $\boldsymbol{I}$ is the second order identity tensor. The linear elasticity law relates the second Piola-Kirchhoff stress tensor $\boldsymbol{S}=J_{e} \boldsymbol{F}_{e}^{-1} \boldsymbol{\sigma}\left(\boldsymbol{F}_{e}^{t}\right)^{-1}$ where $\boldsymbol{\sigma}$ is the stress tensor and the Green-Lagrange strain tensor $\boldsymbol{E}_{e}=1 / 2\left(\boldsymbol{F}_{e}^{t} \boldsymbol{F}_{e}-\boldsymbol{I}\right) ; \boldsymbol{S}=\mathbb{C}: \boldsymbol{E}_{e}$, where $\bullet: \bullet$ is the double inner product. Since an elastoviscoplastic framework is assumed, the plastic strain rate is driven by the resolved shear stress on each slip system $\theta$. The velocity gradient tensor reads

$$
\boldsymbol{L}^{\theta}=\dot{\boldsymbol{F}}^{\theta} \boldsymbol{F}^{\theta-1}=\dot{\gamma}^{\theta} \boldsymbol{s}_{0}^{\theta} \otimes \boldsymbol{n}_{0}^{\theta}
$$

where $\dot{\gamma}^{\theta}$ the resolved shear stress rate on the $\theta^{\text {th }}$ slip system. The orientation of the lattice is important with respect to the loading direction. Thus, for $\Theta$ slip systems the velocity gradient tensor reads

$$
\boldsymbol{L}=\sum_{\theta=1}^{\Theta} \dot{\gamma}^{\theta} \boldsymbol{s}_{0}^{\theta} \otimes \boldsymbol{n}_{0}^{\theta}=\sum_{\theta=1}^{\Theta} \dot{\gamma}^{\theta} \boldsymbol{P}_{0}^{\theta}
$$


where $\boldsymbol{P}_{0}^{\theta}=s_{0}^{\theta} \otimes \boldsymbol{n}_{0}^{\theta}$ is the so-called non-symmetric Schmid tensor. The decomposition of the deformation gradient rate tensor becomes

$$
\boldsymbol{L}=\boldsymbol{L}_{e}+\boldsymbol{F}_{e} \boldsymbol{L}_{p} \boldsymbol{F}_{e}^{-1}
$$

where $\boldsymbol{L}_{e}$ and $\boldsymbol{L}_{p}$ are the elastic and plastic gradient rate tensors. To complete the constitutive description of the crystalline material, the plastic slip rates 2.10 rely on the resolved shear stress. The relationship between the resolved shear stress $\tau^{\theta}$ and the shear rate $\dot{\gamma}^{\theta}$ reads

$$
\dot{\gamma}^{\theta}=\left\langle\frac{\left|\tau^{\theta}-c x^{\theta}\right|-\tau_{0}}{K}\right\rangle^{n} \eta^{\theta}
$$

where $\tau_{0}$ is the critical resolved shear stress, $c$ is the hardening coefficient, $(K, n)$ are material constants related to Norton law, $\eta^{\theta}=\operatorname{sign}\left(\tau^{\theta}-c x^{\theta}\right)$ and $\langle\bullet\rangle$ are Macauley brackets. Furthermore, the isotropic hardening behavior is not investigated, i.e., only the kinematic hardening behavior is considered while assuming an exponential behavior

$$
\dot{x}^{\theta}=\dot{\gamma}^{\theta}-d\left|\dot{\gamma}^{\theta}\right| x^{\theta}
$$

where $d$ is a material parameter. Several slip systems may lead to the same plastic strain rate, thus the growth of the resolved shear stresses $\tau^{\theta}$ is governed by a phenomenological hardening law. From Equations (2.13), and 2.14), and by applying the consistency condition

$$
\dot{x}^{\theta}=\frac{1}{c} \sum_{\kappa} \boldsymbol{H}_{\theta \kappa}\left|\dot{\gamma}^{\kappa}\right|
$$

where $\left[\boldsymbol{H}_{\theta \kappa}\right]$ is the interaction matrix, $\delta_{\theta \kappa}$ is the Kronecher operator and $H_{\theta \kappa}=$ $c\left(\eta^{\kappa}-d x^{\kappa}\right) \delta_{\theta \kappa}$ the hardening moduli of the interaction matrix. Table 3 gathers 
all model parameters and their initial values chosen based on the IF literature 31,32 .

\subsection{2. $C++$ implementation}

One of the main challenges is the coupling between experimental and numerical data in a single framework. An in-house $\mathrm{C}++$ code dealing with all the facets of the problem from the acquired height profile images to the identification of parameters while computing the mechanical responses has been designed. Regarding the FE computations (performed in Code_Aster [33]), a C++ routine has been written to automatically generate the input files with all the necessary data, i.e., the microstructure, the boundary conditions, the mesh, and the constitutive parameters. Simulations are automatically started and the results are sent back to the main code to solve the identification problem iteratively. This main code deals, with $i$ ) the surface profile images in height levels, $i i$ ) the $2 \mathrm{D}$ mesh (in pixels) related to the global DIC formulation, iii) the $3 \mathrm{D}$ mesh (in $\mathrm{mm}$ ) used to solve the finite element problem, and $i v$ ) the $2 \mathrm{D}$ mesh (in pixels) where the sensitivity fields are projected on the DIC matrix to solve the Integrated DIC problem. As proposed in Ref. [21], the parameterization is based on a log scale of the ratio between the current value of the parameter and its initial guess. It is defined such that the sought parameters in the new setting $\{\boldsymbol{q}\}$ are expressed from the initial basis as $\{\boldsymbol{q}\}=\log \left\{\boldsymbol{p} / \boldsymbol{p}_{\mathbf{0}}\right\}$, where $\{\boldsymbol{p}\}$ is the vector gathering the values of the parameters and $\left\{\boldsymbol{p}_{0}\right\}$ their initial values.

As a key feature, the measurement, the computation, and the identification steps rely on a 3D surface formulation where the out-of-plane displacements are 
also used to identify the micromechanical behavior of crystals. The simulations are performed on the measured 3D microstructures with their lattice orientation. Figure 5(a) shows the finite element model of the microstructure where the two grains are modeled with their lattice orientations (with the corresponding colors in the $\{001\}$ pole figure shown in Figure 21.

Furthermore, the applied boundary conditions are obtained from the displacements measured via DIC. The numerical simulations being 3D, the displacement of the triangular boundaries are obtained assuming a linear interpolation between the edge $L^{+}$and $L^{-}$(i.e., intersection between the measured surface $\left(S^{+}\right.$and $\left.S^{-}\right)$and the triangular boundary surface) and the apex $\left(P^{+}\right.$ and $P^{-}$) where its displacement is equal to the mean value of the corresponding edge (respectively $L^{+}$and $L^{-}$).

To conclude, a regularization method 34 is implemented to prevent meaningless identification when the influences of material parameters on the observables are weak. Therefore, a functional $\left(\chi_{R}\right)$ is associated with the identification functional $\left(\bar{\chi}_{f}\right)$ to enable for the changes of the sought parameters only if their corresponding sensitivities are higher than a specific bound. $\chi_{R}^{2}$ is a convex function reaching its minimum equal to zero and reads

$$
\chi_{R}^{2}=\{\boldsymbol{p}\}\left[\boldsymbol{C}_{p}^{R}\right]^{-1}\{\boldsymbol{p}\}
$$

where $\left[\boldsymbol{C}_{p}^{R}\right]$ is the covariance matrix of the material parameters. The regularized functional is written as $\chi_{f}^{2}=\bar{\chi}_{f}^{2}+\chi_{R}^{2}$ because both functionals are dimensionless 
and normalized to one. Finally, the regularization of the linear system reads

$$
\left([\boldsymbol{M}]_{I D I C}+\lambda^{*}[\boldsymbol{I}]\right)\{\delta \boldsymbol{p}\}=\{\boldsymbol{b}\}+\lambda^{*}\left(\left\{\boldsymbol{p}_{0}\right\}-\{\boldsymbol{p}\}\right)
$$

where $\lambda^{*}$ is the regularization parameter whose choice is performed to cancel out the influence of noise 34 .

\section{Experimental results}

320 profile images are acquired and 8-bit encoded such that the total range is used over the entire images set. The ratio between $\mathrm{mm}$ and pixels is equal to $110 \mathrm{~nm} /$ pixel, and the ratio between $\mathrm{mm}$ and height levels is constant and equal to $6.5 \mathrm{~nm} /($ digital level). Figure 6 shows the reference image and the last image of the experiment after the application of the bias correction.

Figure 7 shows a Backscattered Electron (BSE) image of the $\mu$-specimen and its surrounding area where slip bands are revealed due to Electron Channelling Contrast. Furthermore plasticity has occurred during the experiment, as expected.

\subsection{D-DIC measurements}

Figure 8 (a) shows the $u_{Y}$ component of the displacement field obtained with the 3D-DIC algorithm at the last time step. The corresponding mesh is also shown. Because the displacements are very small and the specimen area contains only a limited amount of pixels $(120 \times 40$ pixels $)$, the accuracy of the measured displacements over time is limited. Therefore, a coarse mesh is used to increase 
the robustness of the DIC procedure. Figure 8(b) shows the displacement amplitude $\left(u_{Y}^{+}(t)-u_{Y}^{-}(t)\right)$ between the upper constrained surface $\left(S^{+}\right)$and the lower one $\left(S^{-}\right)$where $\gamma_{u}=26 \mathrm{~nm}$ is the standard displacement uncertainty. The signal possesses a notable amount of noise, which is challenging for further investigations. Figure 8 (b) also shows the prescribed displacement at the boundary of the macroscopic specimen. The ratio between the two displacements (of the order of 5,000$)$ is constant, as expected. The distance between the two grips is $32 \mathrm{~mm}$ and the $\mu$-specimen length $12 \mu \mathrm{m}$ is equal to $3.8 \times 10^{-4}$. However, the ratio experimentally observed is equal to $2 \times 10^{-4}$ and corresponds to a strain of the $\mu$-specimen of $1.6 \%$. This results shows that the microscopic strain is not equal to the global macroscopic strain. It originates from microscopic heterogeneities or small discontinuities of the macroscopic geometry.

Figure 9 shows the digital level residual fields reconstructed at the last time step of the experiment and the last iteration of the DIC procedure. The latter is obtained by computing the difference between each pixel of the reference image and the corrected deformed image [29] and decreases by about $16 \%$. The gain originates from the vertical displacement determination that affects the entire ROI.

The main issue (see Figure 9 ) is that the signal to noise ratio is very small. To overcome this challenge a new strategy is proposed, $i$ ) piecewise-linear time steps are assumed for each loading phase and for each considered surfaces $\left(S^{+}\right.$and $S^{-}$) for the components $u_{Y}$ and $u_{Z}$ (see Figure 10 in red), ii) the displacements of the apex (lines $L^{+}$and $L^{-}$) of both boundary surfaces are identified with 
integrated 3D-DIC, and iii) the measured macroscopic loads are adapted to fit the section of the microscopic specimen and some material parameters are identified based on the minimization of the combination of the loads and image residuals. The $u_{X}$ component is not considered because of its larger noise level.

With this pre-processing strategy, two objectives have already been fulfilled, namely, $i$ ) the displacement fields have been measured over time and $i i$ ) the micromechanical plastic behavior and its evolution have been observed. The remaining objectives to achieve are $i$ ) the coupling between experimental and numerical solutions (with the selected crystal plasticity law), ii) the identification of the displacement of the apex of the boundary triangles and iii) the identification of the constitutive parameters.

\section{Boundary conditions and material parameter identification}

The identification is investigated with the 3D model and the measured microstructure. First, the in-depth displacements at the constrained boundaries are assessed. Four unknowns, namely $\lambda_{Y}^{+}$, and $\lambda_{Z}^{+}, \lambda_{Y}^{-}$, and $\lambda_{Z}^{-}$are applied as an amplitude correction to $u_{Y}^{+}$and $u_{Z}^{+}$at point $P^{+}$(apex of $S^{+}$), and to $u_{Y}^{-}$ and $u_{Z}^{-}$at point $P^{-}$(apex of $S^{-}$, see Figure 11$)$.

\subsection{Sensitivity analysis}

A sensitivity analysis is carried out and the uncertainty is evaluated with the covariance matrix $\left[\boldsymbol{C}^{p}\right]_{I D I C}$ of the unknowns $[22$. A $2 \%$ perturbation on each parameter is applied to compute the sensitivity fields. The vector of kine-

matic and material parameters reads $\{\boldsymbol{p}\}=\left\{\lambda_{Y}^{+}, \lambda_{Z}^{+}, \lambda_{Y}^{-}, \lambda_{Z}^{-}, \tau_{0}, c, d\right\}$. Figure 
12(a) shows the kinematic Hessian for the entire loading history. The most influent parameters are $\lambda_{Y}^{+}$and $\lambda_{Y}^{-}$whereas the material parameters exhibit the smallest influences. This result shows that accurate identification of the boundary conditions is of paramount importance prior to any analysis of the material parameters. Figure 12 (b) shows the corresponding correlation matrix. The high level of anti-correlation between the parameters $(c, d)$ will impact their identifications.

\subsection{Identification}

The underlying boundary conditions are now determined with IntegratedDIC by solving iteratively Equation 2.17). Figure 13(a) shows the change of the four kinematic parameters and Figure 13 (b) shows that of the functional $\chi_{f}$. The converged solution is reached in 10 iterations. Furthermore, three kinematic parameters, namely $\lambda_{Y}^{-}, \lambda_{Z}^{+}$, and $\lambda_{Z}^{-}$converged to a close solution with respect to their initial values. However, $\lambda_{Y}^{+}$varies by about $20 \%$, which means that a significant shear strain occurs on the subsurface of the $\mu$-specimen during the experiment.

Table 4 gathers the measured values and compares the dimensionless residuals between the initial 3D-DIC solution, with more kinematic freedom and the integrated DIC solution, with only few degrees of freedom associated with boundary conditions. Therefore, the small residual increase is regarded as a validation of the integrated approach.

The monitored macroscopic loads are scaled to the specimen section and a combined image and load identification scheme is used, namely IF-DIC. The 
global cost function reads

$$
\bar{\chi}^{2}=\frac{N_{f}}{1+N_{f}} \bar{\chi}_{f}^{2}+\frac{1}{1+N_{f}} \chi_{F}^{2}
$$

where the weight of the two functionals originates from a Bayesian foundation [21] to account in a fair manner for both sources of information. $\chi_{F}^{2}$ is the load residual

$$
\chi_{F}^{2}=\frac{1}{\gamma_{F}^{2} N_{t}}\left\{\boldsymbol{F}_{m}-\boldsymbol{F}_{c}\right\}^{t}\left\{\boldsymbol{F}_{m}-\boldsymbol{F}_{c}\right\}
$$

where $N_{t}$ is the number of time step, $\left\{\boldsymbol{F}_{m}\right\}$ and $\left\{\boldsymbol{F}_{c}\right\}$ the vectors gathering the measured and computed loads, respectively, and $\gamma_{F}=5.97 \times 10^{-6} \mathrm{~N}$ is the standard load uncertainty [27]. The same choice of regularization methodology is made and the global functional reads $\chi^{2}=\bar{\chi}^{2}+\chi_{R}^{2}$. As a first step, only the material parameters are sought, while the boundary conditions are the previously assessed ones. Figures 14 (a) and 14(b) show the changes of the material parameters and residuals. The global residual $(\chi)$ decreases by about $1 \%$ and stabilizes after 4 iterations. Furthermore, while the load residual $\left(\chi_{F}\right)$ is significantly minimized the kinematic residual remains approximately constant. Figure 14(c) shows the experimental, initial, and final computed load histories. Table 5 gathers the constitutive parameters at convergence. Up to $50 \%$ changes are observed with respect to the initial guess. The load residual has been decreased by $25 \%$, which proves that it is sensitive to the sought parameters. However, its final value is still large, which is an indication of a model error associated with the constitutive law. Last, no changes in the kinematic parameters are obtained when a new identification is performed with the assessed 
material parameters. Reflecting on the influence of the unknown boundary conditions on the crystal plasticity parameter identification, one may consider a direct tensile test of a micro-specimen, e.g., by employing the nano-force tensile stage proposed by Bergers et al. [27] to provide relevant data to perform a precise identification with accurate measurements of the load history at the scale of the $\mu$-specimen. Such a change of the methodology would, however, come at the expense of a more complex experimental procedure.

\section{Conclusion}

The investigation and the characterization of the micromechanical behavior of a micro-beam made of two grains have been achieved. On the experimental side, two new procedures have been proposed, $i$ ) the design of a triangular prism shape micro-specimen with a known microstructure embedded in a macrospecimen and $i$ ) the use of a global DIC algorithm to measure the 3D surface displacement field. On the identification side, a novel strategy has been designed to overcome the encountered high noise level on the height profile images. The latter has prevented direct approaches such as Finite Element Method Updating to succeed because the initial 3D global DIC measurement is too noisy. The choice was made to use an integrated approach to work directly on the images but the scatter on the boundary conditions prevented a direct evaluation of the sensitivity fields.

Based on this limitation, a two-step identification strategy was adapted, $i$ ) estimation of the boundary conditions and ii) determination of the constitu- 
tive parameters. The boundary conditions were assumed to be piece-wise linear with time. First, the identification via integrated-DIC was performed only with the height profile images to assess the underlying boundary conditions. Subsequently, the macroscopic loads were adapted to the specimen section and both sources of information were combined to assess the sought constitutive parameters, which describe the kinematic hardening relationship in the crystal plasticity material model.

The concept proposed herein is very general and can be applied to any micromechanical experiment aiming to characterize a constitutive law describing a micro-mechanical behavior. While optical profilometry brings the advantage of 3D displacement fields, other imaging systems can also be use such as scanning electron microscopy or regular optical microscopy depending on the sought models and the observation scale. The technique gives new insights into the micro-mechanical behavior with the combination of images and loads based on a methodology that can cope with high noise levels.

\section{Acknowledgment}

MB thanks Professor Marc G.D. Geers for giving him the opportunity to join the Mechanics of Material research group to perform the experiments reported herein. The authors acknowledge the help of Dr. Hugo Leclerc in the implementation of the 3D-DIC code. This work was supported by the Région Ile de France and BPI France (DICCIT and THERMOFLUIDE-RT projects). 


\section{Bibliography}

[1] J. D. Eshelby, The determination of the elastic field of an ellipsoidal inclusion, and related problems, Series A, Mathematical and Physical Sciences 241 (1957) 376-396.

[2] Z. Hashin, S. Shtrikman, A variational approach to the theory of the elastic behaviour of multiphase materials, Journal of the Mechanics and Physics of Solids 11 (2) (1963) 127 - 140.

[3] R. Hill, Continuum micro-mechanics of elastoplastic polycrystals, Journal of the Mechanics and Physics of Solids 13 (2) (1965) $89-101$.

[4] P. Franciosi, A. Zaoui, Multislip in f.c.c. crystals a theoretical approach compared with experimental data, Acta Metallurgica 30 (8) (1982) 1627 1637.

[5] A. M. Cuitino, M. Ortiz, Computational modelling of single crystals, Modelling and Simulation in Materials Science and Engineering 1 (3) (1993) 225.

[6] L. Tabourot, M. Fivel, E. Rauch, Generalised constitutive laws for f.c.c. single crystals, Materials Science and Engineering: A 234â236 (0) (1997) $639-642$.

[7] D. Raabe, M. Sachtleber, Z. Zhao, F. Roters, S. Zaefferer, Micromechanical and macromechanical effects in grain scale polycrystal plasticity experimentation and simulation, Acta Materialia 49 (17) (2001) 3433 - 3441. 
[8] A. Musienko, A. Tatschl, K. Schmidegg, O. Kolednik, R. Pippan, G. Cailletaud, Three-dimensional finite element simulation of a polycrystalline copper specimen, Acta Materialia 55 (12) (2007) 4121 - 4136.

[9] R. Martins, C. Ohms, K. Decroos, Full 3d spatially resolved mapping of residual strain in a 316L austenitic stainless steel weld specimen, Materials Science and Engineering: A 527 (18 - 19) (2010) 4779 - 4787.

[10] T. Hoc, J. Crépin, L. Gélébart, A. Zaoui, A procedure for identifying the plastic behavior of single crystals from the local response of polycrystals, Acta Materialia 51 (18) (2003) 5477 - 5488.

[11] O. Dmitrieva, P. W. Dondl, S. Maoeller, D. Raabe, Lamination microstructure in shear deformed copper single crystals, Acta Materialia 57 (12) (2009) $3439-3449$.

[12] C. Tasan, J. Hoefnagels, M. Geers, Microstructural banding effects clarified through micrographic digital image correlation, Scripta Materialia 62 (11) (2010) $835-838$.

[13] D. Raabe, M. Sachtleber, H. Weiland, G. Scheele, Z. Zhao, Grain-scale micromechanics of polycrystal surfaces during plastic straining, Acta Materialia 51 (6) (2003) $1539-1560$.

[14] E. Héripré, M. Dexet, J. Crépin, L. Gélébart, A. Roos, M. Bornert, D. Caldemaison, Coupling between experimental measurements and polycrystal finite element calculations for micromechanical study of metallic materials, International Journal of Plasticity 23 (9) (2007) 1512 - 1539. 
[15] K. T. Kavanagh, R. W. Clough, Finite element applications in the characterization of elastic solids, International Journal of Solids and Structures 7 (1) (1971) $11-23$.

[16] C. Tasan, M. Diehl, D. Yan, C. Zambaldi, P. Shanthraj, F. Roters, D. Raabe, Integrated experimental-simulation analysis of stress and strain partitioning in multiphase alloys, Acta Materialia 81 (0) (2014) 386 - 400.

[17] A. Guery, F. Latourte, F. Hild, S. Roux, Identification of crystal plasticity law parameters using kinematic measurements in polycrystals, in: WCCM XI - 11th World Congress on Computational Mechanics, Barcelona, Spain, 2014.

[18] L. Méric, G. Cailletaud, Single crystal modeling for structural calculations: Part 2 - finite element implementation, Journal of Engineering Materials and Technology 113 (1991) 171-182.

[19] S. Roux, F. Hild, Digital image mechanical identification (DIMI), Experimental Mechanics 48 (4) (2008) 495-508.

[20] J. Neggers, J. P. M. Hoefnagels, M. G. D. Geers, F. Hild, S. Roux, Timeresolved integrated digital image correlation, International Journal for $\mathrm{Nu}-$ merical Methods in Engineering 103 (3) (2015) 157-182.

[21] M. Bertin, F. Hild, S. Roux, Optimization of a biaxial tensile specimen geometry for the identification of constitutive parameters based upon full field measurements, Strain (2016) . 
[22] M. Bertin, F. Hild, S. Roux, F. Mathieu, H. Leclerc, P. Aimedieu, Integrated digital image correlation applied to elastoplastic identification in a biaxial experiment, The Journal of Strain Analysis for Engineering Design $51(2)(2016)$ 118-131.

[23] S.-H. Lee, H. Utsunomiya, T. Sakai, Microstructures and mechanical properties of ultra low carbon interstitial free steel severely deformed by a multi-stack accumulative roll bonding process, Materials transactions 45 (7) (2004) 2177-2181.

[24] G. Besnard, F. Hild, S. Roux, Finite-element" displacement fields analysis from digital images: Application to Portevin-Le Châtelier bands, Experimental Mechanics 46 (6) (2006) 789-803.

[25] K. Han, M. Ciccotti, S. Roux, Measuring nanoscale stress intensity factors with an atomic force microscope, EPL (Europhysics Letters) 89 (6) (2010) 66003.

[26] J. Neggers, J. Hoefnagels, F. Hild, S. Roux, M. Geers, Direct stress-strain measurements from bulged membranes using topography image correlation, Experimental Mechanics 54 (5) (2014) 717-727.

[27] L. Bergers, J. Hoefnagels, M. Geers, On-wafer time-dependent high reproducibility nano-force tensile testing, Journal of Physics D: Applied Physics $47(49)(2014) 495306$.

[28] D. Lecompte, A. Smits, H. Sol, J. Vantomme, D. V. Hemelrijck, Mixed numerial-experimental technique for orthotropic parameter identification 
using biaxial tensile tests on cruciform specimens, International Journal of Solids and Structures 44 (2007) 1643-1656.

[29] H. Leclerc, J. Périé, S. Roux, F. Hild, Integrated digital image correlation for the identification of mechanical properties, Vol. LNCS 5496, Springer, Berlin, 2009, pp. 161-171.

[30] M. Bertin, C. Brown, A basis and method for removal and replacement of doubtful height observations in topographic measurements, International conference on surface metrology, oral presentation, 2012.

[31] P. Franciosi, Glide mechanisms in b.c.c. crystals: An investigation of the case of $\alpha$-iron through multislip and latent hardening tests, Acta Metallurgica 31 (9) (1983) $1331-1342$.

[32] D. D. Tjahjanto, S. Turteltaub, A. S. J. Suiker, S. van der Zwaag, Modelling of the effects of grain orientation on transformation-induced plasticity in multiphase carbon steels, Modelling and Simulation in Materials Science and Engineering 14 (4) (2006) 617.

[33] H. David, Comportements élastoviscoplastiques mono et polycristallins, R5.03.11 - Code_Aster (2014) 1-58.

[34] R. Gras, H. Leclerc, F. Hild, S. Roux, J. Schneider, Identification of a set of macroscopic elastic parameters in a $3 \mathrm{~d}$ woven composite: Uncertainty analysis and regularization, International Journal of Solids and Structures $55(2015) 2-16$. 
Figures

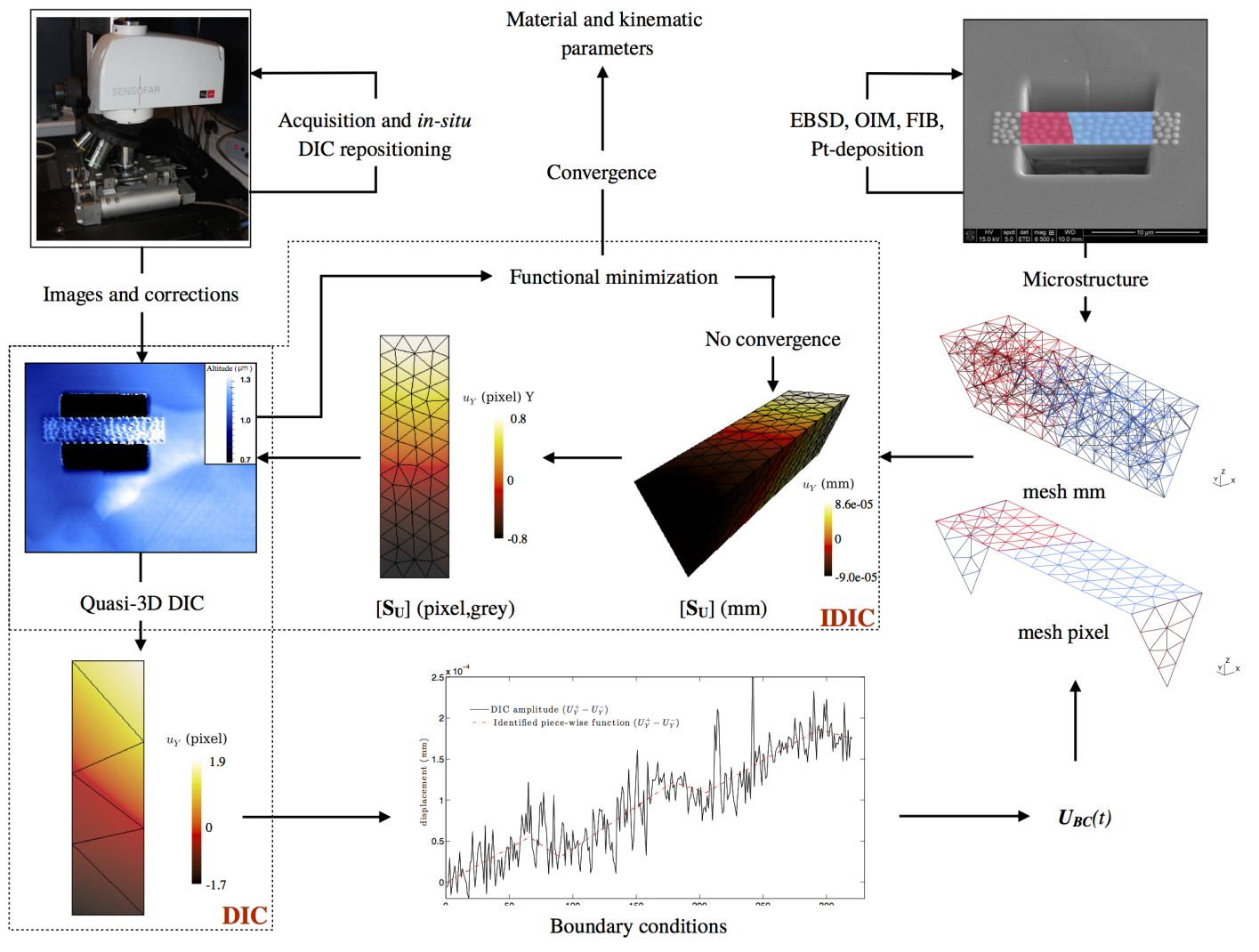

Figure 1: Schematic view of the methods used herein to extract boundary conditions and crystal plasticity parameters. The different steps of this methodology are explain in Section 2 


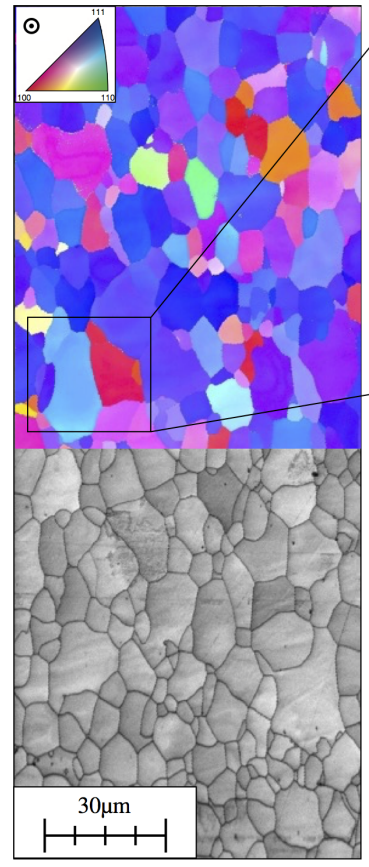

(a)

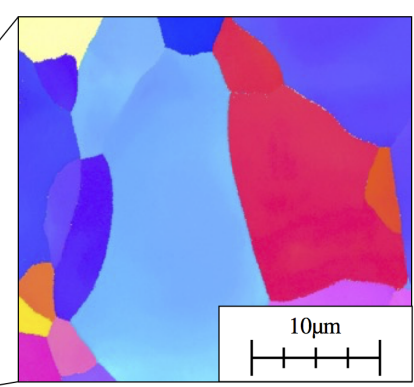

(b)

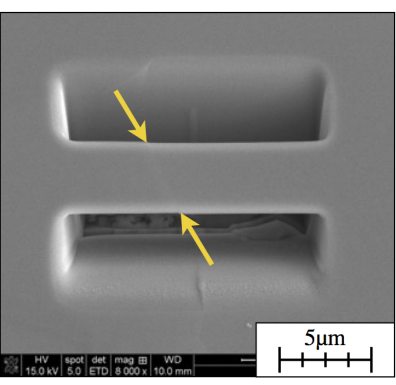

(c)

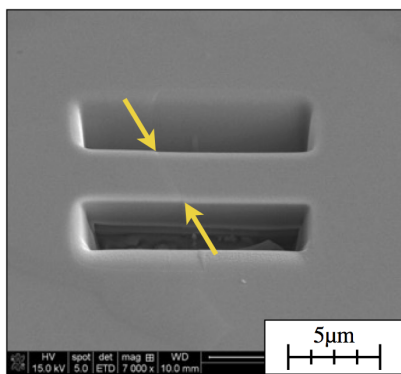

(d)

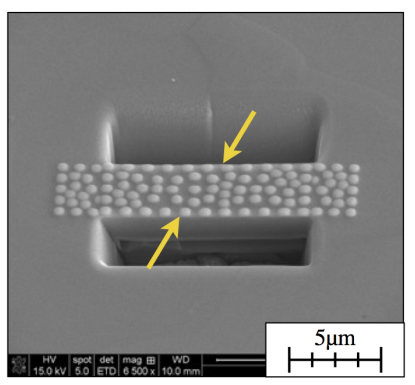

(e)

Figure 2: Image Quality (IQ) map (a) and Inverse Pole Figure (IPF) maps (a,b), resulting from the OIM measurement of the studied IF-steel. The longitudinal direction of the sample is the horizontal direction in all the figures. (b) Chosen location for the $\mu$-specimen and (c) $\mu$-specimen after the FIB process. (d) $\mu$ specimen observed with a tilt of $52^{\circ}$. The underlying microstructure and the grain boundary are observed. (e) $\mu$-specimen after platinum deposition. The arrows depict the location of the grain boundary 

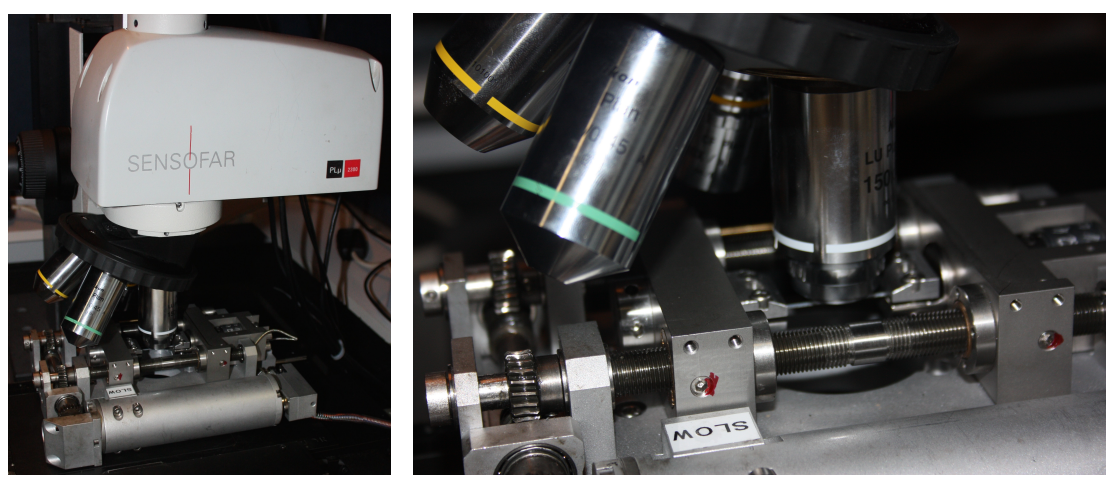

Figure 3: Experimental apparatus with the tensile stage on motorized $(x, y)$ sample positioning stage within a Sensofar optical profiler $(150 \times$ magnification lens) 


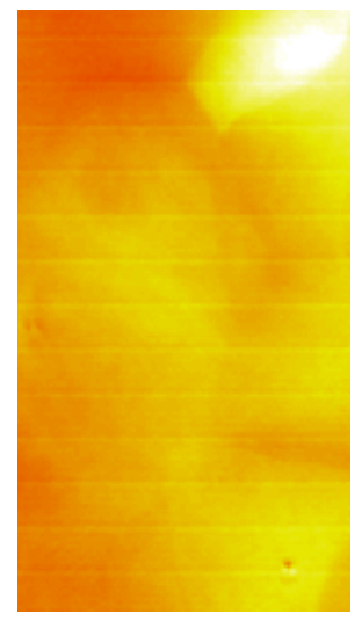

(a)

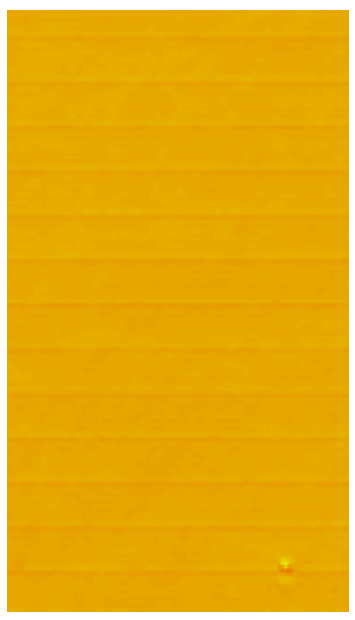

(b)

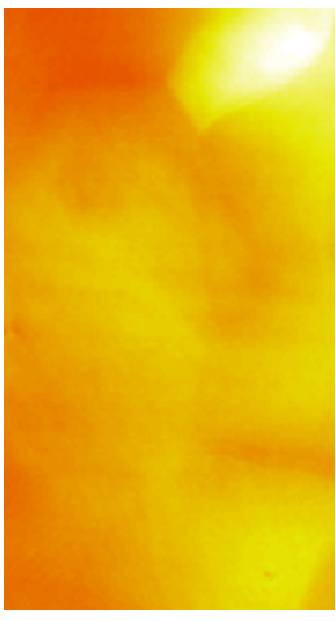

(c)

Figure 4: (a) Systematic errors on a profile image generated by the confocal microscope. Fringes and dust particles are present that are detrimental for the DIC computations. (b) Image correction obtained from the random acquisition of 400 images of a reference surface, and (c) profile image corrected from the systematic bias 


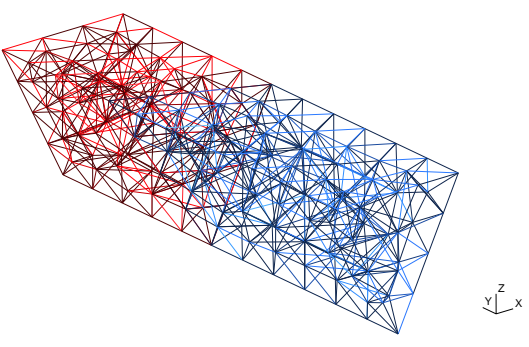

(a)

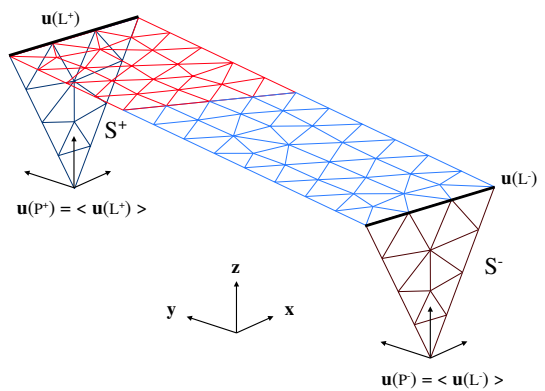

(b)

Figure 5: (a) 3D mesh of the $\mu$-specimen with the two grains visible (two different colors). (b) 2D mesh on the top surface used for IDIC, shown together with the sides where the boundary conditions are interpolated from the initial DIC computation

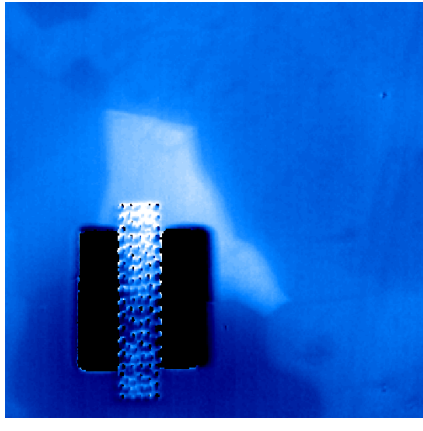

(a)

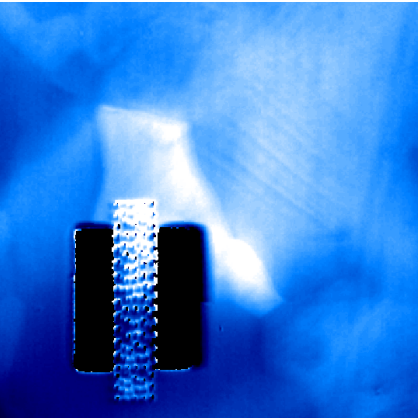

(b)
Altitude $(\mu \mathrm{m})$

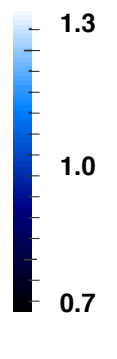

Figure 6: (a) Reference image and (b) image at the last time step of the experiment after application of the systematic error correction 


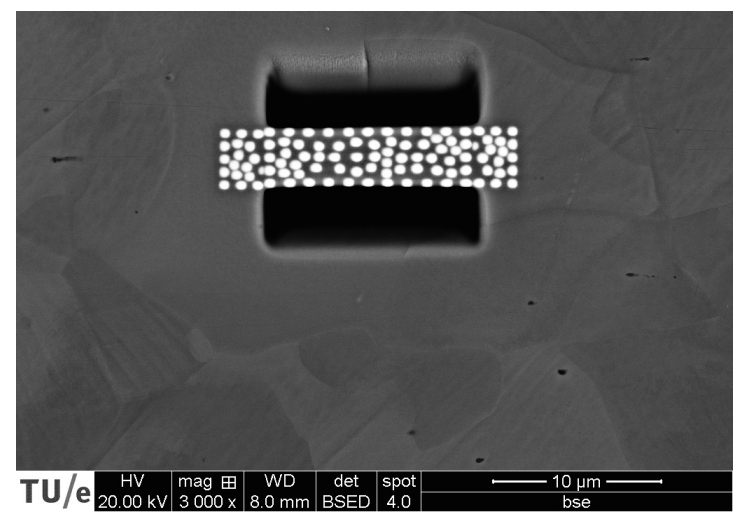

Figure 7: Backscattered electron (BSE) image of a larger view at the end of the experiment. The slip bands are observable around the $\mu$-specimen inside the grains
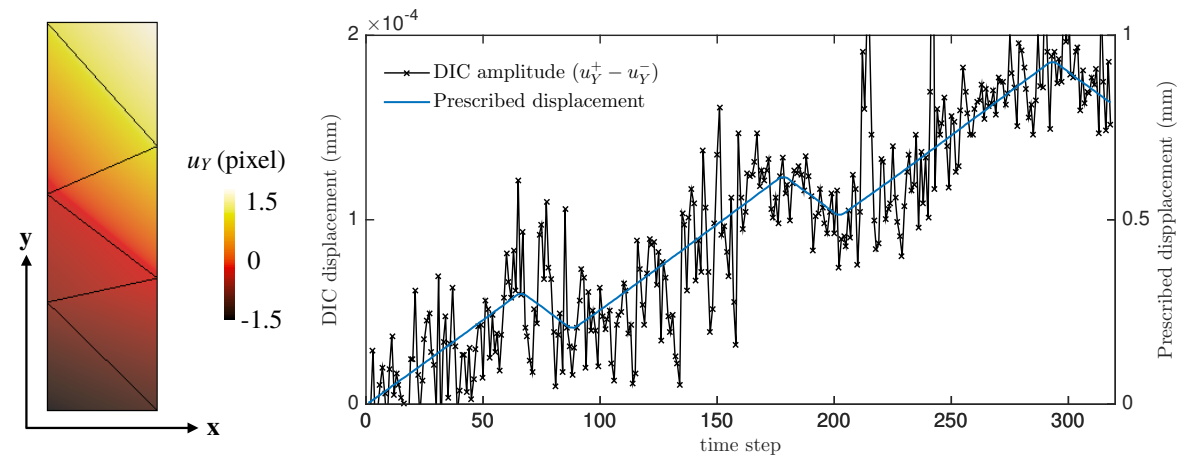

(a)

(b)

Figure 8: (a) Component $u_{Y}$ of the displacement field at the last time step of the experiment evaluated via DIC. (b) Displacement amplitude $\left(u_{Y}^{+}(t)-u_{Y}^{-}(t)\right)$ in the longitudinal direction and prescribed displacement with an adapted scale (see left size of the graph) 


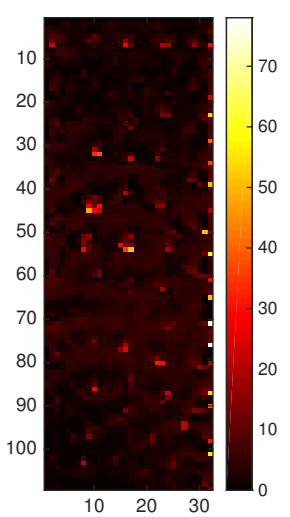

(a) $\chi_{f}=3.9$

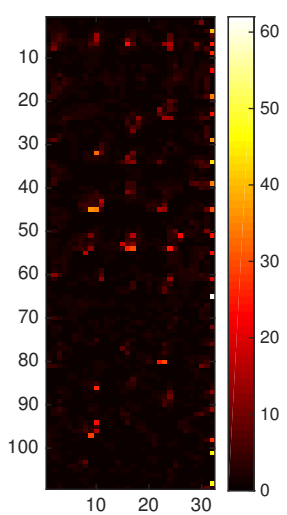

(b) $\chi_{f}=3.2$

Figure 9: (a) Residual field at the last time step of the experiment for the first iteration and (b) at convergence. The fields are expressed in digital levels (i.e., 1 digital level $=6.5 \mathrm{~nm})$

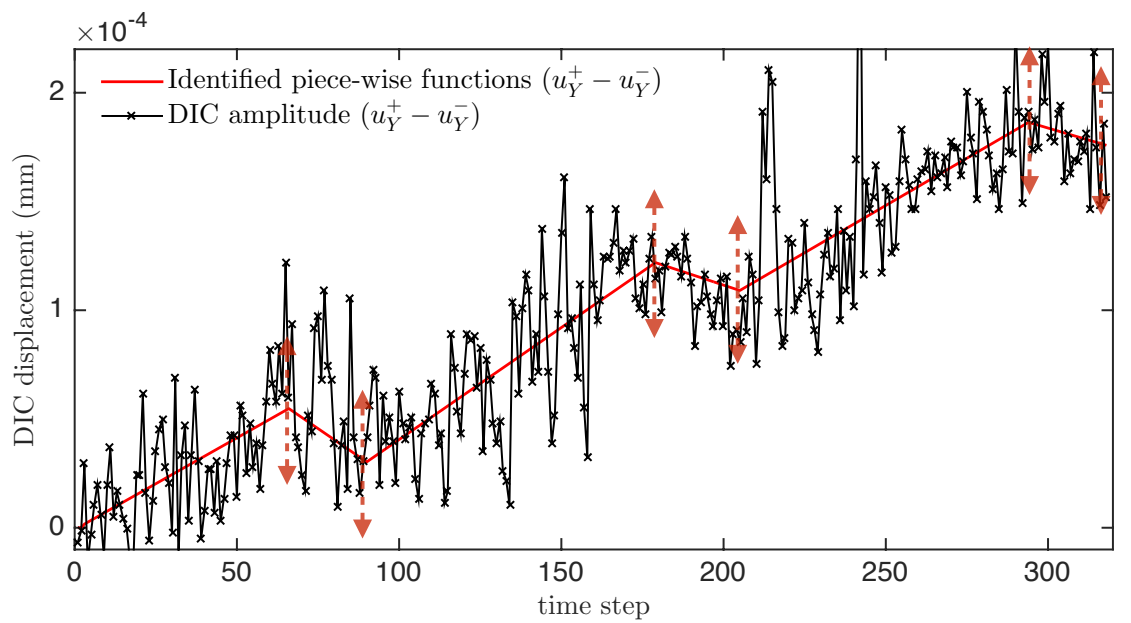

Figure 10: Displacement amplitude $\left(u_{Y}^{+}(t)-u_{Y}^{-}(t)\right)$ in the longitudinal direction from DIC (the mean of the two extreme nodes) results and the corresponding piecewise-linear steps. The arrows denote the considered degrees of freedom 


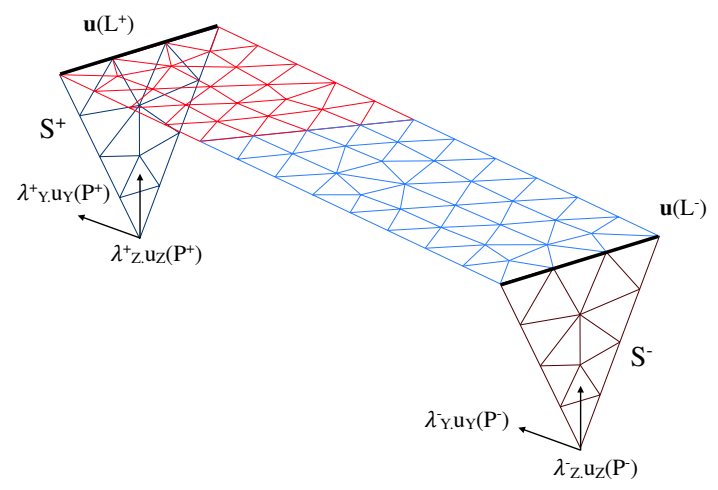

Figure 11: Kinematic unknowns corresponding to the boundary conditions on the bottom part of the specimen

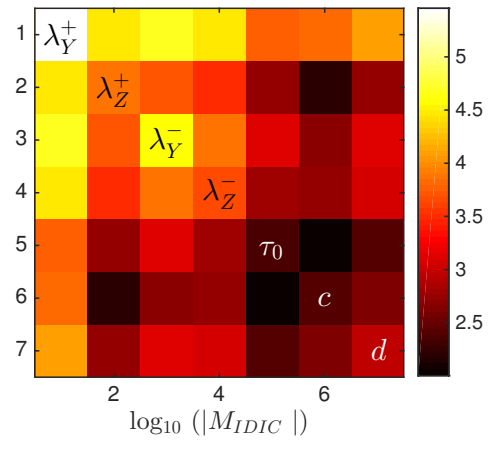

(a)

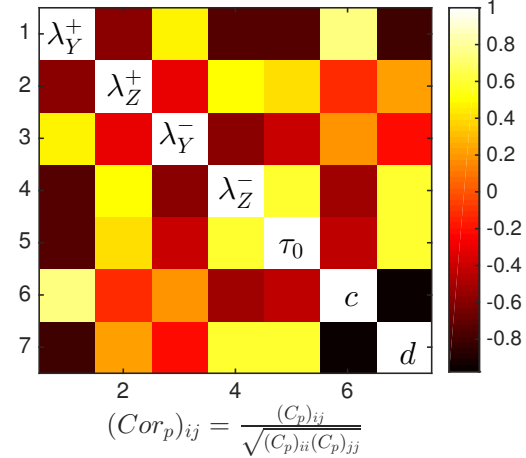

(b)

Figure 12: (a) Kinematic Hessian $[\boldsymbol{M}]_{I D I C}$ and (b) correlation matrix of the kinematic Hessian $[\boldsymbol{M}]_{I D I C}$, both for the entire loading history 

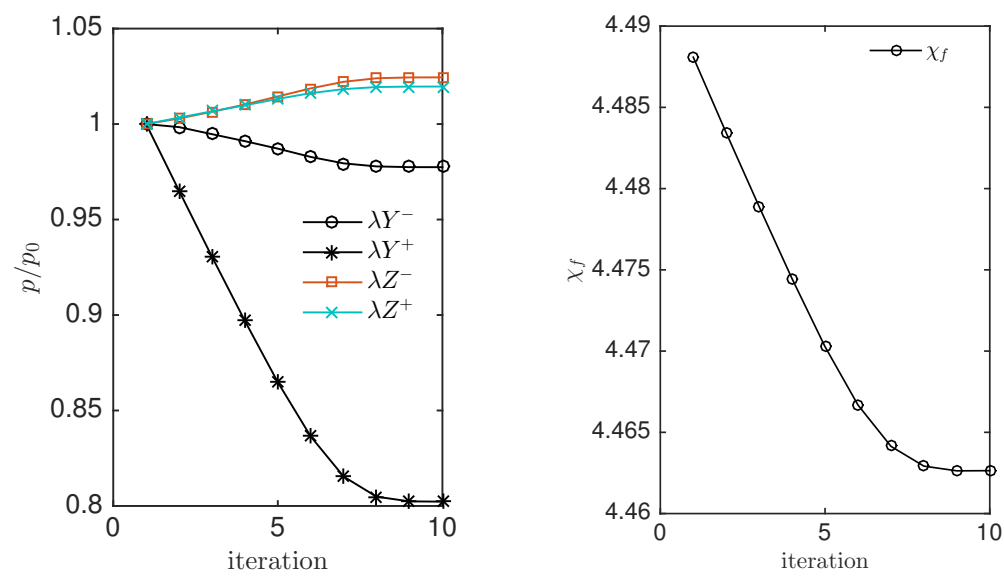

Figure 13: (a) Parameter changes during the iterations of IDIC and (b) evolution of the dimensionless residual

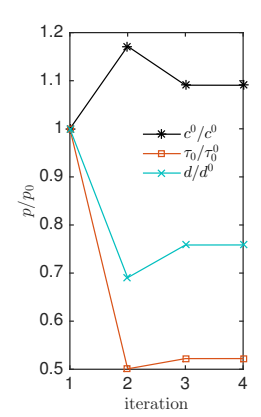

(a)

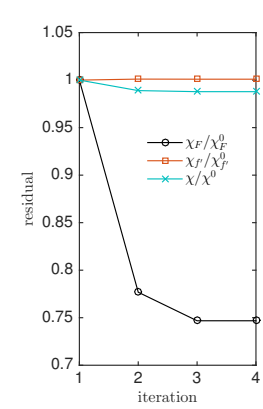

(b)

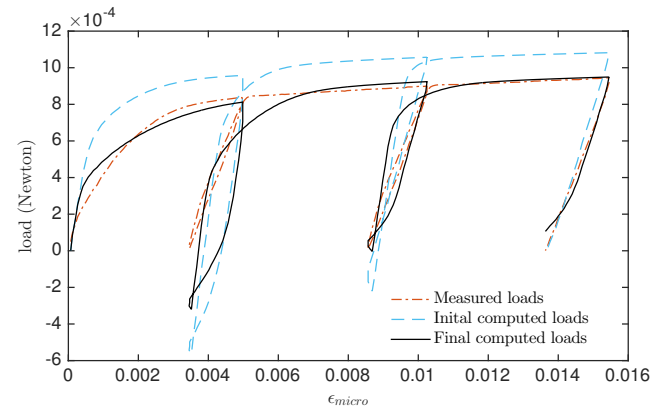

(c)

Figure 14: (a) Material parameters and (b) residuals as functions of iteration number. (c) Reaction forces on the $\mu$-specimen boundaries 


\section{Tables}

Table 1: Chemical composition of the studied IF-steel

\begin{tabular}{|c|cccccccccc|}
\hline Composition & $\mathrm{C}$ & $\mathrm{Mn}$ & $\mathrm{Al}$ & $\mathrm{Ti}$ & $\mathrm{Nb}$ & $\mathrm{Si}$ & $\mathrm{S}$ & $\mathrm{P}$ & $\mathrm{N}(\mathrm{ppm})$ & $\mathrm{Fe}$ \\
\hline (wt \%) & 0.0022 & 0.09 & 0.015 & 0.033 & 0.008 & 0.003 & 0.007 & 0.012 & 28 & bal. \\
\hline
\end{tabular}

Table 2: Euler angles of the two grains measured via OIM and maximum misorientation angle $(\delta)$ within each grain

\begin{tabular}{|l|c|c|c|c|}
\hline & \multicolumn{3}{|c|}{ Euler angle $\left(^{\circ}\right)$} & misorientation $\left(^{\circ}\right)$ \\
\hline Denomination & $\psi$ & $\theta$ & $\phi$ & $\delta$ \\
\hline Grain 1 (red) & 281 & 8 & 47 & 0.70 \\
Grain 2 (blue) & 211 & 45 & 150 & 0.76 \\
\hline
\end{tabular}

Table 3: All parameters of the Meric and Cailletaud crystal plasticity model and their initial values chosen based on the IF literature [31, 32]

\begin{tabular}{|l|c|c|c|c|c|c|c|c|}
\hline Mechanism & \multicolumn{3}{|c|}{ Elasticity } & \multicolumn{2}{c|}{ Viscosity } & \multicolumn{3}{c|}{ Plasticity } \\
\hline Model parameter & $C_{11}$ & $C_{12}$ & $C_{44}$ & $K$ & $n$ & $\tau_{0}$ & $c$ & $d$ \\
\hline Unit & $\mathrm{GPa}$ & $\mathrm{GPa}$ & $\mathrm{GPa}$ & $\mathrm{MPa}$ & $(-)$ & $\mathrm{MPa}$ & $\mathrm{GPa}$ & $(-)$ \\
\hline Initial value & 233.5 & 135.5 & 118 & 12 & 10 & 40 & 40 & 1500 \\
\hline
\end{tabular}


Table 4: Identified values of the kinematic parameters

\begin{tabular}{|l|c|c|c|c|c|}
\hline Name & $\chi_{f}$ & $\lambda_{Y}^{+}$ & $\lambda_{Z}^{+}$ & $\lambda_{Y}^{-}$ & $\lambda_{Z}^{-}$ \\
\hline DIC & 3.7 & - & - & - & - \\
\hline IDIC & 4.5 & 0.81 & 1.02 & 0.98 & 1.02 \\
\hline
\end{tabular}

Table 5: Identified parameters of the crystal plasticity model

\begin{tabular}{|l|c|c|c|c|c|c|}
\hline Name & $\chi$ & $\chi_{f}$ & $\chi_{F}$ & $\tau_{0}(\mathrm{MPa})$ & $c(\mathrm{GPa})$ & $d(-)$ \\
\hline Initial value & 4.64 & 4.46 & 78 & 40 & 40 & 1600 \\
\hline IF-DIC & 4.52 & 4.47 & 58 & 21 & 44 & 1140 \\
\hline
\end{tabular}

